\title{
EL ROL DE LA COMISIÓN DE DEFENSA DE LA CÁMARA DE DIPUTADOS EN LA
} FORMULACIÓN DE LA POLÍTICA DE DEFENSA

\author{
Jaime B Aeza Freer \\ U NIVERSIDAD DE C HILE \\ ANDRÉS D OCKENDORFF V ALDÉS \\ Academia Nacional de Estudios Políticos y Estratégicos de Chile (Anepe) \\ N atalia V argas Palacios \\ U NIVERSIDAD DE C HILE
}

Fecha de recepción: 17 de mayo de 2010.

Fecha de aceptación: 01 de junio de 2010.

\begin{abstract}
Resumen
Este estudio examina el rol de la Comisión de Defensa de la Cámara de Diputados en el proceso de toma de decisión en materias de Seguridad y Defensa (Policy Making Process -PMP- en inglés). En este contexto, las premisas de este estudio guardan relación con las atribuciones de estas comisiones, que van más allá de la mera aprobación presupuestaria y proyectos para convertirlas en ley, sino que, además, otras actividades centrales para los intereses de sus miembros en materias tales como la supervisión de las decisiones del gobierno en materia de Defensa, como asimismo su relación directa con las Fuerzas Armadas, considerando que cada diputado y senador pueden in Quir en el desarrollo de políticas públicas de cada rama castrense.
\end{abstract}

Palabras clave: Comisiones de Defensa, Cámara de Diputados, Fuerzas Armadas-Chile

\begin{abstract}
This research examines the role played by the Defense Committee at the Chilean House of Deputies in regards to the decision making process known Policy Making Process (PMP). In this context, the study is based on the assumption that the Committee has more attributions than producing legislation, budget approving and similar bills, but also other key political activities. These other issues are related to supervising government's decisions in the Defense sector, as well as a direct relationship with each branch of the Armed Forces, being considered the possibility of direct in 凶uence of each Congressman in each of the service.
\end{abstract}

Key words: Defense Committee, House of Deputies, Armed Forces-Chile

Para correo y consultas a Jaime Baeza jbaezaf@u.uchile.cl o Andrés Dockendorff dockendorf@gmail. com. Agradecemos el apoyo \nanciero de ANEPE y especialmente de don Víctor Guzmán Martínez. Este es la versión corregida de un trabajo homónimo presentado ante el IX Congreso Chileno de Ciencia Política, noviembre de 2010. 


\section{Introducción}

Hasta ahora, existen muy pocos estudios acerca de las comisiones de defensa en Chile y, principalmente, de carácter descriptivo. Entre estos ejemplos es posible encontrar el trabajo de Contreras, Polloni y Ortiz (2001) que produjo una visión panorámica acerca de las atribuciones constitucionales y legales que rigen su accionar, aunque desactualizado producto de las reformas constitucionales de 2005. En este mismo sentido, la Red Sudamericana de Seguridad (RESDAL, 2008) produjo un volumen describiendo el funcionamiento y el marco legal de varias comisiones de defensa en el continente sudamericano. Dentro de su trabajo, atención han puesto en las principales características acerca de la periodicidad de las sesiones y cuáles son las atribuciones que diputados y senadores pueden ejercer.

En el contexto mencionado, este estudio innova en términos de proponer nuevas respuestas acerca de los incentivos que entrega el sistema para que los miembros del Congreso quieran ser parte de las comisiones de defensa, y cómo el PMP es desarroIlado, en tanto también analiza las prioridades políticas de sus miembros. A priori, parece ser que más relevante que la mera formulación de proyectos de ley, están más interesados en la supervisión de la política de defensa, modificando los proyectos del Ejecutivo y en interrelación directa con las Fuerzas Armadas.

La experiencia comparada enseña que la capacidad de oversight que tienen las comisiones de defensa sobre las Fuerzas Armadas y el Ejecutivo en otras partes del mundo les permite pasar de un mero contrapeso de poderes, a constituirse en actores fundamentales en la formulación de las políticas de defensa. Para cumplir este fin, se requiere, junto con el conocimiento de la actividad de los miembros de la Comisión, que son especialistas en asuntos legislativos y no necesariamente especialistas en defensa, contar con una cuantificación certera de la actividad realizada en términos de su consecuencias para todo el sector y la población, como asimismo, la indispensable generación de un cuerpo civil de asesores que puedan ayudar de manera profesional en la formulación de dicha política, cuestión clave para una institución como la ANEPE.

\section{Antecedentes para el debate}

El Libro de la Defensa de Chile publicado en 2010 señala que en ambas cámaras del Honorable Congreso Nacional existirán comisiones de defensa, las que tienen entre otras funciones "la de analizar e informar al respectivo plenario todos los proyectos de ley relativos a la defensa nacional, en general, y a las Fuerzas Armadas en particular" (2010: 119). Además, el documento sostiene que las cámaras concurren a la aprobación presupuestaria del sector, amén de poder declararse en estado de asamblea en caso de guerra exterior y, a su vez, el Senado autorizar o denegar la entrada 
de tropas extranjeras al interior de la República, como asimismo, la salida de tropas chilenas al exterior (Ibíd.: 119).

Si bien este documento oficial da cuenta de la labor en términos formales de las actividades de las comisiones de Defensa de la Honorable Cámara de Diputados y del Honorable Senado de la República, pareciera ser definiciones de orden más bien jurídico formal, en tanto lo que determinan la Constitución Política de la República, como asimismo otras instituciones legales tales como la Ley Orgánica del H. Congreso Nacional, entre otras. Sin embargo, esta definición dice poco acerca de la vida interna de ambas comisiones y cuál es su verdadera influencia en la construcción y funcionamiento de la política nacional de defensa. Si bien la vida diaria de las comisiones pasa también por lo Legislativo, este proyecto está interesado en la actividad prelegislativa y no legislativa que realiza y que impacta en el desarrollo de la actividad de seguridad y defensa del país, englobando dicha tarea en el concepto de Policy Making Process (PMP). Junto con el gobierno y los partidos políticos, el cuerpo legislativo es un actor crucial del PMP, ya que se espera que capten las demandas, problemas y necesidades de los ciudadanos, formulando y/o aprobando políticas ad hoc (Stein et al., 2006; Stein y Tommasi, 2006). PMP en democracia.

Los sistemas de formulación de políticas públicas -que reconoce crecientemente complejos- están asociados íntimamente a la gobernabilidad democrática (Peters, 2003). El estudio comparado de Formulación de Políticas Públicas (PFP) ha sido promovido por la línea de investigación desarrollada por el Banco Interamericano de Desarrollo (BID). Esta preocupación se ha traducido en diversos documentos de trabajo y publicaciones. El libro La Política de las Políticas Públicas (2006) constituye el esfuerzo más acabado por relevar, a escala comparada, la importancia de estudiar este asunto en América Latina. En ese trabajo se define el PFP como;

"Un proceso de negociaciones e intercambios (o transacciones) entre los actores políticos", que "(...) engloba todo el proceso de discusión, aprobación e implementación de las políticas públicas" (Stein et al., 2006: 18).

Diversos actores o jugadores participan interactivamente del PFP en distintos escenarios formales (legislatura y gabinete) e informales (Stein et al., 2006: 18). El enfoque del BID resalta la correlación positiva que existiría entre la calidad de las políticas y los resultados (outcomes), con la capacidad de los actores para conseguir acuerdos y basar las políticas públicas en la cooperación. De la calidad del PFP de cada país dependerán: la estabilidad, adaptabilidad, coherencia, coordinación, la calidad de la implementación, la eficiencia y la orientación hacia el interés público de las políticas (Stein et al., 2006: 17). En esta perspectiva, la calidad de las políticas públicas (variable dependiente) estaría afectada por la habilidad de los actores políticos para promover y obtener resultados basados en consensos (Spiller, Stein y Tommasi, 2003). 
El estudio comparado del proceso de formulación de políticas públicas (PFP) ha sido promovido por la línea de investigación desarrollada por el Banco Interamericano de Desarrollo (BID), una preocupación que se ha traducido en diversos documentos de trabajo y publicaciones. El libro La Política de las Políticas Públicas (2006) constituye el esfuerzo más acabado por relevar, a escala comparada, la importancia de estudiar los PFP en América Latina. En este trabajo se define el PFP como: "un proceso de negociaciones e intercambios (o transacciones) entre los actores políticos", que "(...) engloba todo el proceso de discusión, aprobación e implementación de las políticas públicas" (Stein et al., 2006: 18).

Diversos actores o jugadores participan interactivamente del PFP en distintos escenarios formales (legislatura, gabinete) e informales (Stein et al., 2006: 18). El enfoque del BID resalta la correlación positiva que existiría entre la calidad de las políticas y los resultados (outcomes) con la capacidad de los actores para conseguir acuerdos y fundamentar las políticas públicas en la cooperación. De la calidad del PFP de cada país dependerán la estabilidad, adaptabilidad, coherencia, coordinación, la calidad de implementación, la eficiencia y la orientación hacia el interés público de las políticas (Stein et al., 2006: 17). Lo anterior está ligado a la capacidad de los actores (o jugadores) para obtener acuerdos de calidad que sean estables en el tiempo (Spiller, Stein y Tommasi, 2003). Desde esta perspectiva, la calidad de las políticas públicas (variable dependiente) estaría afectada por la habilidad de los actores políticos para promover y obtener outcomes basados en la cooperación y el consenso (Spiller, Stein y Tommasi, 2003).

Lo anterior no es exclusivo del enfoque del BID y de las conclusiones de los trabajos de Stein y Tommasi (2006), Spiller et al., (2003), Aninat, Londregan et al., (2006) y otros, sino que es recogido por estudiosos que han seguido de cerca procesos de formulación de políticas públicas desde la asesoría de políticas públicas en el caso chileno. Entre ellos, Eugenio Lahera tiene un juicio coincidente, ya que, en su opinión, las políticas públicas "(...) basadas en discusiones con todos los actores relevantes se acercarán más a los objetivos de bien público, al mismo tiempo tendrán el necesario apoyo sostenido de la ciudadanía. Podrán a la vez ser más comprehensivas y viables" (Lahera 2002: 31). PFP en Chile: teoría y evidencia.

En el trabajo del BID el caso chileno es destacado por la calidad de sus políticas públicas, junto a los casos brasileño y uruguayo. El informe del organismo analiza un conjunto de variables que incidirían para que en Chile exista una eficaz formulación de las políticas públicas. El trabajo desarrollado por Aninat, Londregan et al. (2006) apunta en el mismo sentido. Existen incentivos para la cooperación y la transacción en el circuito legislativo y en el proceso de políticas públicas en base a acuerdos que mejorarían la calidad de las políticas (Aninat, Londregan, Navia y Vial 2006; Aninat 2004; Boeninger 2008; Stein et al., 2006). Esta perspectiva recoge la visión promovida por los trabajos y estudios patrocinados por el BID (Stein et al., 2006; Stein y Tommasi, 2006; Spiller et al., 2003, Aninat, Londregan et al., 2006). 
La combinación de un Ejecutivo con amplia capacidad de establecer agenda y actores con capacidad de veto incentivaría la cooperación, la negociación y el acuerdo en la formulación de las políticas (Aninat 2004; Stein et al., 2006; Aninat, Londregan et al., 2006). El proceso de generación, articulación y formulación de políticas en Chile estaría determinado, entre otros factores, por la negociación y un alto grado de consenso sobre las políticas, donde el proceso de formulación de políticas se desarrolla a través de acuerdos y negociaciones entre el Ejecutivo y la oposición, lo que responde también a ciertas restricciones institucionales (por ejemplo: quórums, sistema electoral) y a la necesidad dotar de legitimidad el proceso de "hechura" de las políticas (Schmidt 2007).

De acuerdo al trabajo del BID (2006) y Aninat (2004), Chile es el país mejor evaluado de la región en cuanto a las características de sus políticas públicas (estabilidad, adaptabilidad, entre otras). A pesar del enorme poder asignado por la Constitución de la República al Presidente, el sistema chileno de formulación de políticas opera sobre la base de un conjunto de jugadores con capacidad de veto ${ }^{2,3}$. Existiría una secuencia de negociaciones y acuerdos a partir de los cuales se discuten y negocian las políticas (Stein et al., 2006; Aninat 2004).

\section{Comisiones de defensa: experiencia comparada}

Las comisiones de defensa cumplen un rol que va más allá de lo meramente legislativo. En este sentido, vale la experiencia a nivel comparado. En el caso de los regímenes parlamentarios europeos, los que en general comparten ciertas características acerca de su funcionamiento. En este sentido, un rol relevante es el concepto anglosajón de oversight en tanto Born lo considera como la permanente supervisión que realiza acerca del funcionamiento del gobierno y, en este caso, del manejo del sector defensa, la gobernanza como medida de asegurar las formas democráticas en el manejo del sector defensa y el control del sector, tanto en lo reactivo (fiscalizador) como asimismo proactivo (generación de normas que permitan el mejor funcionamiento del sector (Born, 2002: 4). La actividad de oversight no puede ser solo del Poder Ejecutivo, también el Congreso y en especial la comisión respectiva debe estar presente (USAID, 2005). Esta es una característica esencial en un sistema democrático. Como el propio autor suizo reconoce, Hitler y Stalin tenían control sobre las Fuerzas Armadas de su poder, pero carecían de todo control democrático (Born, 2002: 5). Este último punto es crucial, ya que la literatura reconoce que las relaciones cívico-militares ya no se basan únicamente en el control, sino que también en la efectividad y la eficiencia democrática del mismo (Bruneau y Matei, 2008; Bruneau, Matei y Sakoda, 2009), enfoque

2 Sobre los jugadores con veto y su impacto en el proceso político democrático véase Tsebelis (2002)

3 Sobre los jugadores con veto y su impacto en el proceso político democrático véase Tsebelis (2002). 
bajo el cual la efectividad de la supremacía civil no se mide únicamente a partir de la presencia/ausencia de posibilidad de levantamientos militares, sino a partir del rol que juegan las autoridades civiles en la formulación y definición de las políticas de defensa (Fuentes, 2008), espacio donde los congresos y sus comisiones especializadas resultan claves (Bruneau, 2005; Born, Flury y Lunn, 2003).

Entre las actividades que se encuentran en los sistemas parlamentarios europeos, Born reconoce al menos las siguientes características propias de comisiones de defensa, tales como realizar informes que involucren el parecer de los partidos de oposición o minoritarios, aprobar y modificar los proyectos de legislación que tengan que ver con seguridad y defensa, recibir información y canalizar las inquietudes en la materia que tenga la sociedad civil organizada.

La realidad europea responde a regímenes parlamentarios donde el propio gobierno reside en el Parlamento. En los regímenes presidenciales, como el nuestro, el gobierno está radicado en sede diferente del Congreso Nacional, lo que hace que además de representar las visiones de la oposición y otros grupos al interior del Parlamento, en los sistemas como el chileno la comisión cumple una función política de check and balances, de acuerdo al modelo presidencialista. En ese sentido, a pesar de la naturaleza reactiva de muchas legislaturas de América Latina, los estudiosos reconocen que las comisiones pueden hacer la diferencia en las legislaturas (Cox y Morgenstern, 2002; Morgenstern, 2002; Rivera, 1998), en la medida en que pueden afectar la estructura de incentivos (junto a la variable reelección) de las relaciones Ejecutivo-Legislativo y la capacidad de los congresos para influir en el PMP (Bejar, 2007; Morgenstern, 2002).

El Congreso chileno combina instancias de veto con otras de cooperación y negociación (Boeninger, 2007; Aninat, Londregan, Navia y Vial, 2006; Aninat, 2006; Schmidt Hebbel, 2007). En contra de algunos diagnósticos comparados excesivamente formales sobre las relaciones Ejecutivo-Legislativo en el caso chileno, la evidencia empírica sugiere que el Congreso chileno tiene una creciente influencia en el proceso decisorio (Boeninger, 2007; Huneeus y Berríos, 2004; Berríos y Gamboa, 2006; Aninat, 2006), lo que se ha ganado gracias a la mayor especialización y profesionalización y la estructuración de sistemas de asesorías competentes (Aninat, 2006; Boeninger, 2007), pero por sobre todo, al eficiente funcionamiento del sistema de comisiones, cuestión desconocida por la ciudadanía (Boeninger, 2007).

A pesar de que es débil si se lo compara con el Congreso de Estados Unidos y su poderoso sistema de comisiones, "(...) the chilean Congress is unusually profesional and technically competent by Latin American standars" (Aninat, Londregan, Navia y Vial, 2006: 31). Las comisiones legislativas juegan un rol clave en la formulación de gran parte de la legislación más relevante (Carey, 2002; Siavelis, 2002). Este aspecto, junto con la profesionalización de los legisladores y las capacidades técnicas del conjunto de la legislatura, permiten a John Carey concluir que: 
"(...) the Chilean Congress is highly professionalized- particularly for a young legislature- and that its committee system is accumulating substantial expertise, and is organized and dominated by the majority coalition. Within the comparative study of legislatures, these are all regarded as indicators of strong legislative capacity" (Carey, 2002: 222- 253).

Por otro lado, al existir correspondencia entre los ámbitos de actividad estatal y la estructura de comisiones, el grado de coordinación del Ejecutivo y el Legislativo es alto, especialmente en perspectiva comparada (Aninat, 2006; Aninat, Londregan, Navia y Vial, 2006; Stein y Tommasi, 2006; Stein et al., 2006).

La importancia de las comisiones permanentes en el sector defensa es discutido por algunos estudiosos, quienes plantean que las comisiones de defensa de ambas cámaras son entes más bien decorativos, producto de la poca preparación de sus miembros en los temas de defensa y un sistema de asesoría poco especializado en estas materias (Weeks, 2006; Valdivieso, 2009). Este trabajo pretende revisar estas conclusiones y, eventualmente, discutirlas a partir de la evidencia empírica.

De acuerdo a una corriente de la literatura, la seguridad y defensa no constituirían una prioridad para los políticos de América Latina, caracterizándose por un menor nivel de escrutinio público y debate que otras políticas públicas (Pion-Berlin y Trinkunas, 2007). Lo anterior se explicaría en función de patrón histórico asociado a Fuerzas Armadas con débiles capacidades y que se constituyen más en una amenaza para los gobiernos que para los vecinos, un entorno de relativa tranquilidad regional y la baja importancia que le asignarían los votantes (y por ende los políticos y legisladores) a la provisión de la defensa nacional (Pion-Berlin y Trinkunas, 2007). Junto a ello, existe la creencia equivocada de que las políticas de seguridad y defensa son una tarea que recae "naturalmente" en el Ejecutivo, visión desmentida por los casos históricos en Estados democráticos (Born, Fluir y Lunn, 2003). Por otra parte, en el campo de la defensa, las tareas convencionales de control, discusión y debate sectorial que ejercen las legislaturas, especialmente a través de las comisiones, se ven complejizadas producto de la considerable especialización que requieren, la naturaleza de la información que se maneja, entre otras (Van Eekelen, 2005).

El papel que desempeñan los congresos en la defensa nacional cobra mayor relevancia al asumir que las relaciones cívico militares (CMR) en la actualidad no se basan solo en el control, sino que también en la efectividad y la eficiencia (Bruneau y Matei, 2008; Bruneau, Matei y Sakoda, 2009)4. Si bien Bruneau, Matei y Sakoda

Al respecto, y en un análisis a escala del Cono Sur, Fuentes (2008) sostiene que la efectividad de la supremacía civil no se puede definir a partir de la presencia/ausencia de posibilidad de levantamientos militares, sino que términos de las definiciones constitucionales sobre el rol de las Fuerzas Armadas, el rol que juegan las autoridades civiles en la formulación y definición de las políticas de defensa y las transformaciones de la estructura de la defensa (Fuentes, 2008). 
(2009) argumentan que los Consejos de Seguridad Nacional (CSN) pueden jugar un rol clave al favorecer las relaciones cívico-militares en estas dimensiones (control, efectividad y eficiencia), en este trabajo se aborda los desafíos en materia de CMR poniendo atención en el rol de la legislatura, punto que reconoce Thomas Bruneau cuando señala que las legislaturas sirven como soporte para la trilogía control; efectividad y eficiencia, ya que

"(...) they ensure democratic civilian control by maintaining real separation of powers, controlling the budget, and excersing oversight. Diversity of political representation through elections, and the development of expertise among members and particularly their staffs, allows legislatures to improve the effectiveness of roles and missions (...) Furthermore, legislatures routinely implement an oversight function through hearings, auditing units, and inspectors general to ensure efficiency" (Bruneau, 2005: 124).

En general, en perspectiva comparada, el Poder Legislativo juega un rol central cuando se trata de establecer la supremacía civil y garantizar la gobernabilidad democrática (Born, Flury y Lunn, 2003). "Las ventajas de un accionar fluido entre el Parlamento y el sector de la defensa, debe ser vista también en lo que atañe a la implementación de normas e instancias de gestión y relacionamiento con las fuentes militares, en términos de información y comunicación para el cumplimiento de las tareas legislativas, fiscalizadoras e investigadoras, en beneficio de una mejor capacidad de gestión, control y legislación en las áreas de defensa, seguridad y agenda de política exterior" (Celi, 2003: 7). En otras palabras, la relación entre la legislatura y las Fuerzas Armadas, constituye "(...) una condición necesaria para el ejercicio democrático de su control y conducción desde políticas consensuadas y socialmente asumidas" (Celi, 2003: 7).

Todo lo anterior va ligado al papel que desempeñan, en perspectiva comparada, y cómo se desarrollarán en este las comisiones de defensa de los congresos o parlamentos.

\section{Las comisiones de defensa y su rol en democracia}

La experiencia de los congresos de América Latina indica que "(...) las comisiones hacen la diferencia en las legislaturas" (Rivera, 1998: 531), ya que afectarían "(...) la estructura de incentivos que acota el desarrollo del proceso legislativo" (Bejar, 2007: 103), en tanto son "(...) una de las variables internas más importantes que pueden determinar la capacidad de las legislaturas para influir en la elaboración de las políticas gubernamentales" (Rivera, 1998: 565). En ese sentido, "las comisiones cumplen un papel cada vez más importante en el proceso legislativo, tanto como espacio de discusión como de mejora técnica" (García y Sánchez, 2002: 33). Pero su rol no solo afecta el policy making, sino que son instrumentos ad hoc para supervisar y fiscalizar, así como para "(...) Ilamar la atención del gobierno sobre temas clave que 
pudieran no estar incluidos en la agenda gubernamental" (Rivera, 1998: 566). Esta influencia aumenta y es mayor en el caso de las comisiones permanentes y correspondientes a la estructura administrativa (Olson y Norton 1996, citado en Rivera, 1998) ${ }^{5}$.

En el caso del campo de la defensa y la seguridad, Born (2002) sostiene que, considerando la complejidad del sector, una estructura de comisiones bien desarroIlada es necesaria si el Poder Legislativo espera tener influencia real en la administración (Born, 2002).

Este ejercicio parte reconociendo que el rol de los congresos en América Latina no suele incorporarse al debate académico (Donadio, 2004). De todas formas, un diagnóstico positivo señala que las comisiones de defensa se han ido constituyendo en un aporte a las CMR, en la medida en que operan como un espacio para que interactúen civiles y militares (Follietti y Tibiletti, 2004). En la región "(...) las comisiones de defensa se convierten en el referente por excelencia al querer conjugar parlamento y defensa" (Follietti y Tibiletti, 2004: 10). "Las comisiones parlamentarias de defensa son una instancia muy importante en cuanto al procesamiento, investigación, análisis, posicionamiento y asesoría sobre la problemática", a pesar de que la responsabilidad recae en todos los legisladores (Celi, 2004: 28).

En algunos países de América Latina no existe una comisión legislativa específica que trate las materias del sector defensa. "Es una práctica común en algunos parlamentos de la región que los asuntos de seguridad y defensa sean tratados por diversos tipos de comisiones, denominadas de seguridad nacional, de Fuerzas Armadas, de asuntos internacionales, de relaciones exteriores, con lo cual se vuelven imprecisas sus funciones y se debilita el posicionamiento y el tratamiento particular de la problemática" (Celi, 2004: 29). Al apreciar las disposiciones formales establecidas en la Constitución de países como Venezuela, Bolivia, Perú y Colombia, Rial (2004) concluye que a pesar de que "(...) el predominio civil en la conducción de la defensa nacional muestra avances, (....) el tratamiento de los congresos es mínimo" (Rial, 2004: 16). En los casos señalados "Suele ser más clara la atribución de potestades al Ejecutivo, pero aun así permanece un gran margen de discrecionalidad", asociado a dos cuestiones básicas: la capacidad efectiva con que cuenta los ejecutivos en materia de políticas de defensa y relaciones cívico-militares y el papel real que tienen las legislaturas en el proceso político de estos países (Rial, 2004: 16).

\footnotetext{
Alemán y Pachón (2008) argumentan, a partir del estudio comparado de los casos de Chile y Colombia, que las comisiones de conciliación (Comisión Mixta en la nomenclatura legal chilena) tienen una "posición privilegiado desde la que influyen en el contenido de las propuestas de ley", ya que rara vez sus propuesta son votadas negativamente (p. 3).
} 
Tabla No 1

Comisiones de Defensa en América Latina

\begin{tabular}{|c|c|c|c|}
\hline País & Cámara de Diputados & Senado & Unicameral \\
\hline Argentina & $\begin{array}{l}\text { Comisión de Defensa } \\
\text { Nacional }\end{array}$ & $\begin{array}{l}\text { Comisión de Defensa } \\
\text { Nacional }\end{array}$ & \\
\hline Bolivia & $\begin{array}{l}\text { Comisión de Defensa y } \\
\text { Fuerzas Armadas }\end{array}$ & $\begin{array}{l}\text { Comisión de Gobierno, } \\
\text { Defensa, Policía Nacional } \\
\text { y Lucha contra el tráfico } \\
\text { ilícito de drogas. }\end{array}$ & \\
\hline Brasil & $\begin{array}{l}\text { Comisión de Relaciones } \\
\text { Exteriores y Defensa } \\
\text { Nacional }\end{array}$ & $\begin{array}{l}\text { Comisión de Relaciones } \\
\text { Exteriores y Defensa } \\
\text { Nacional }\end{array}$ & \\
\hline Chile & $\begin{array}{l}\text { Comisión de Defensa } \\
\text { Nacional }\end{array}$ & $\begin{array}{l}\text { Comisión de Defensa } \\
\text { Nacional }\end{array}$ & \\
\hline Colombia & Comisión Segunda & Comisión Segunda & \\
\hline El Salvador & & & Comisión de Defensa \\
\hline Guatemala & & & $\begin{array}{l}\text { Comisión de Defensa } \\
\text { Nacional }\end{array}$ \\
\hline Honduras & & & $\begin{array}{l}\text { Comisión de Defensa } \\
\text { Nacional }\end{array}$ \\
\hline México & $\begin{array}{l}\text { Comisión de Defensa } \\
\text { Nacional }\end{array}$ & $\begin{array}{l}\text { Comisión de Defensa } \\
\text { Nacional }\end{array}$ & \\
\hline Nicaragua & & & $\begin{array}{l}\text { Comisión de la Paz, } \\
\text { Defensa, Gobernación y } \\
\text { Derechos Humanos }\end{array}$ \\
\hline Paraguay & $\begin{array}{l}\text { Comisión de Defensa } \\
\text { Nacional, Seguridad y } \\
\text { Orden Interno }\end{array}$ & $\begin{array}{l}\text { Comisión de Asuntos } \\
\text { Constitucionales, De- } \\
\text { fensa Nacional y Fuerza } \\
\text { Pública }\end{array}$ & \\
\hline Perú & & & $\begin{array}{l}\text { Comisión de Defen- } \\
\text { sa Nacional, Orden } \\
\text { Interno, Inteligencia, } \\
\text { Desarrollo Alternativo y } \\
\text { Lucha contra las Drogas }\end{array}$ \\
\hline $\begin{array}{l}\text { República } \\
\text { Dominicana }\end{array}$ & $\begin{array}{l}\text { Comisión de Seguridad y } \\
\text { Defensa Nacional }\end{array}$ & $\begin{array}{l}\text { Comisión de Defensa y } \\
\text { Seguridad Nacional }\end{array}$ & \\
\hline Uruguay & $\begin{array}{l}\text { Comisión de Defensa } \\
\text { Nacional }\end{array}$ & $\begin{array}{l}\text { Comisión de Defensa } \\
\text { Nacional }\end{array}$ & \\
\hline Venezuela & & & $\begin{array}{l}\text { Comisión de Defensa y } \\
\text { Seguridad }\end{array}$ \\
\hline
\end{tabular}

Fuente: RESDAL 2008. Atlas Comparativo de la Defensa en América Latina. www.resdal.org. 


\section{Funcionamiento y rol de las comisiones de defensa en Chile}

\section{Las comisiones permanentes: estructura y funciones}

De acuerdo al Reglamento de la Cámara de Diputados, existen tres tipos de comisiones: permanentes, unidas, especiales, mixtas e investigadoras. Existen 24 comisiones permanentes ${ }^{6}$, cuya composición representa a la proporción de diputados de cada partido político.

En el funcionamiento del Congreso, las comisiones legislativas de la Cámara de Diputados son grupos de trabajo conformados por 13 diputados cuyo objetivo: "(...) es permitir el estudio detallado de los proyectos de ley y demás materias sometidas a conocimiento de la Corporación, usualmente se recibe la opinión de expertos en la materia de que se trate y se ofrecen audiencias a organizaciones de la sociedad civil interesadas en el tema" ( $\underline{w w w . b c n} . \underline{\text { cl }})$. Las comisiones reciben los proyectos de ley y las materias que correspondan a su especialidad. Para dicho efecto "podrán solicitar la comparecencia de funcionarios que estén en condiciones de ilustrar sus debates y hacerse asesorar por cualquier especialista en la materia respectiva y solicitar informar u oír a las instituciones y personas que estime conveniente" (Reglamento de la Cámara de Diputados, p. 51). La relación de las comisiones con la ciudadanía está formalizada en la figura de las jornadas temáticas.

La discusión de los proyectos de ley (mensajes y mociones) se realiza en las comisiones permanentes de la Cámara de Diputados y el Senado, en correspondencia a las áreas o campos de la actividad gubernamental. Los proyectos son discutidos en general y en particular bajo un riguroso análisis de los legisladores de la comisión. En este proceso, la comisión invita a las sesiones de la misma a expertos y representantes del Ejecutivo relacionados con la materia en discusión. Los miembros de la comisión pueden presentar indicaciones modificatorias, pero siempre en materias que no sean de iniciativa exclusiva del Presidente y en referencia a las ideas matrices del proyecto en cuestión (Boeninger, 2007: 65). En ese sentido, la mayor parte del trabajo legislativo se realizaría en las comisiones legislativas, donde se votan artículo por artículo los proyectos, luego de que es aprobada la idea de legislar (Boeninger, 2007). Al existir correspondencia entre los ámbitos de actividad estatal y la estructura de comisiones,

6 De Gobierno Interior y Regionalización; de Relaciones Exteriores, Asuntos Parlamentarios e Integración Latinoamericana; de Constitución, Legislación y Justicia; de Educación, Deportes y Recreación, de Hacienda, de Defensa Nacional, de Obras Públicas, Transporte y Telecomunicaciones; de Agricultura, Silvicultura y Desarrollo Rural; de Recursos Naturales, Bienes Nacionales y Medio Ambiente; de Salud; de Trabajo y Seguridad Social; de Minería y Energía; de Economía, Fomento y Desarrollo; de la Vivienda y Desarrollo Urbano, de Derechos Humanos, Nacionalidad y Ciudadanía, de Familia, de Ciencias y Tecnología, de Pesca, Acuicultura e Intereses Marítimos, de la Micro, Pequeña y Mediana Empresa, de Zonas Extremas, de Seguridad Ciudadana, de la Cultura y de las Artes, de la Superación de la Pobreza, Planificación y Desarrollo Social. 
el grado de coordinación del Ejecutivo y el Legislativo es alto, especialmente en perspectiva comparada (Aninat, 2006; BID, 2006).

A través de sus respectivos reglamentos la cámara baja y el Senado dan forma al sistema de comisiones permanentes a las que les corresponde el trabajo de informar los proyectos de ley que la sala somete a su consideración (LOC del Congreso Nacional, Art. 17). Las dos cámaras cuentan con una Comisión de Hacienda, que debe conocer todos los proyectos que involucren componentes de materia presupuestaria o financiera.

¿Qué importancia tiene el sistema de comisiones dispuesto en la institucionalidad legislativa chilena? La discusión de los proyectos de ley (mensajes y mociones) se realiza en las comisiones permanentes de la Cámara de Diputados y el Senado, en correspondencia a las áreas o campos de la actividad gubernamental. Los proyectos son discutidos en general y en particular bajo un riguroso análisis de los legisladores de la comisión. En este proceso, la comisión invita a las sesiones de la misma a expertos y representantes del Ejecutivo relacionados con la materia en discusión (Boeninger, 2007: 65).

Los miembros de la Comisión pueden presentar indicaciones modificatorias, pero siempre en materias que no sean de iniciativa exclusiva del Presidente y en referencia a las ideas matrices del proyecto en cuestión (Boeninger, 2007: 65). La Ley Orgánica Constitucional del Congreso Nacional establece que los proyectos de ley que estén en su primer o segundo trámite constitucional, así como las observaciones realizadas por el Presidente de la República, deben ser informadas por la comisión permanente respectiva (Art. 21). Las comisiones reúnen la información que requieren para discutir los proyectos, para lo cual podrán requerir la asistencia de funcionarios que puedan "(...) ilustrar sus debates, hacerse asesorar por cualquier especialista en la materia respectiva y solicitar informes u oír a las instituciones y personas que estime conveniente" (LOC Congreso Nacional, Art. 22).

De acuerdo al Reglamento de la Cámara de Diputados, existen diferentes tipos de comisiones: permanentes, unidas, especiales, mixtas e investigadoras. Las comisiones especiales son creadas por acuerdo de la Cámara y tienen carácter temporal, formándose para estudiar una legislación o proyecto específico o recopilar información necesaria. Las comisiones investigadoras son organismos colegiados para el ejercicio de las funciones fiscalizadoras de la Cámara de Diputados, cuya competencia será fijada en su acuerdo constituyente, el que además establecerá el plazo que le corresponderá trabajar (Reglamento de la Cámara de Diputados, Art 297-300). Las comisiones unidas corresponden al trabajo conjunto de dos o más comisiones, que podrán abocarse unidas al examen de un asunto determinado (Reglamento de la Cámara de 
Diputados, Art 228). Existen 24 comisiones permanentes ${ }^{7}$, cuya composición representa a la proporción de diputados de cada partido político. Los miembros específicos de las comisiones son elegidos por la Cámara luego de una propuesta realizada por la mesa.

Las comisiones permanentes de la Cámara de Diputados son grupos de trabajo conformados por 13 diputados cuyo objetivo: “(...) es permitir el estudio detallado de los proyectos de ley y demás materias sometidas a conocimiento de la Corporación, usualmente se recibe la opinión de expertos en la materia de que se trate y se ofrecen audiencias a organizaciones de la sociedad civil interesadas en el tema" (www.bcn.cl). Las comisiones reciben los proyectos de ley y las materias que correspondan a su especialidad. Para dicho efecto, "podrán solicitar la comparecencia de funcionarios que estén en condiciones de ilustrar sus debates y hacerse asesorar por cualquier especialista en la materia respectiva y solicitar informar u oír a las instituciones y personas que estime conveniente" (Reglamento de la Cámara de Diputados, Art. 217). La relación de las comisiones con la ciudadanía está formalizada en la figura de las jornadas temáticas.

Por su parte, el Senado cuenta con 20 comisiones permanentes ${ }^{8}$. Las comisiones permanentes de la cámara alta, al igual que las especiales, estarán compuestas por 5 senadores. Cada uno de los 38 miembros del Senado debe pertenecer al menos a una de las 20 comisiones permanentes. Se podrán encomendar el estudio de un asunto o proyecto a dos o más comisiones. Tanto los proyectos de ley, ya sea que estén en primer o segundo trámite constitucional, como las observaciones del Presidente de la República, deberán pasar a la comisión respectiva (Reglamento del Senado, Art. 27-36).

7 De Gobierno Interior y Regionalización; de Relaciones Exteriores, Asuntos Parlamentarios e Integración Latinoamericana; de Constitución, Legislación y Justicia; de Educación, Deportes y Recreación, de Hacienda, de Defensa Nacional, de Obras Públicas, Transporte y Telecomunicaciones; de Agricultura, Silvicultura y Desarrollo Rural; de Recursos Naturales, Bienes Nacionales y Medio Ambiente; de Salud; de Trabajo y Seguridad Social; de Minería y Energía; de Economía, Fomento y Desarrollo; de la Vivienda y Desarrollo Urbano, de Derechos Humanos, Nacionalidad y Ciudadanía, de Familia, de Ciencias y Tecnología, de Pesca, Acuicultura e Intereses Marítimos, de la Micro, Pequeña y Mediana Empresa, de Zonas Extremas, de Seguridad Ciudadana, de la Cultura y de las Artes, de la Superación de la Pobreza, Planificación y Desarrollo Social.

8 De Gobierno, Descentralización y Regionalización; de Relaciones Exteriores; de Constitución, Legislación, Justicia y Reglamento; de Economía; de Hacienda; de Educación, Cultura, Ciencia y Tecnología; de Defensa Nacional; de Obras Públicas; de Agricultura; de Medio Ambiente y Bienes Nacionales; de Trabajo y Previsión Social; de Salud; de Minería y Energía; de Vivienda y Urbanismo; de Transporte y Telecomunicaciones; de Derechos Humanos, Nacionalidad y Ciudadanía; de Intereses Marítimos, Pesca y Agricultura; de Régimen Interior; Revisora de Cuentas; de Ética y Transparencia del Senado. 


\section{La Comisión de Defensa de la Cámara de Diputados en el periodo 2006-2010}

"La existencia de un fuerte presidencialismo no impide la existencia de una relación fluida y constructiva entre las ramas de las Fuerzas Armadas con el Congreso. En efecto, al revisar las normas en esta materia, aparece con nitidez un conjunto de alternativas procesales que permiten establecer adecuados lazos entre los entes, los que permiten al poder legislativo cumplir con las labores para las que fue creado, y dentro de los parámetros normales en los que se inscribe una sociedad democrática" (Polloni, Contreras y Ortiz, 2001: 63). Esta fluidez que existiría en cuanto a los intercambios entre el Poder Legislativo y las Fuerzas Armadas se diluye cuando entramos en el campo de la asignación de los recursos. En cuanto a la discusión presupuestaria, "(...) la labor del Congreso se ve limitada a un rol prácticamente notarial. Otra está marcada por la falta de atribuciones del Congreso en materia de disponer de las fuerzas de aire, mar y tierra, organizarlas y distribuirlas de acuerdo con las necesidades de la seguridad nacional, aspecto que la Constitución entrega en forma exclusiva al Presidente" (Polloni et al., 2001: 71).

Gregory Weeks sostiene de que a pesar de que tanto la Cámara de Diputados como el Senado en Chile tienen comisiones de Defensa:

"(..) they are neither well staffed not particularly influential. There is no permanent staff anywhere within the congressional structure with the expertise on defense issues. Any member of Congress without prior knowledge of such issues must find his or her own resources, and in practice relatively few members do so" (Weeks, 2006: 34).

En general, la asamblea chilena jugaría un rol particularmente decorativo en la formulación de las políticas de relaciones exteriores y defensa (Valdivieso, 2009), donde el control de la agenda sería aún mayor. "Al contrario de lo pasa en otras áreas, la relación del Ejecutivo con la Comisión de Defensa es ocasional, y no cuenta con mecanismos institucionalizados de conexión con el parlamento". Mientras que el Ministerio de Relaciones Exteriores cuenta con una oficina de enlace parlamentario, el Ministerio de Defensa no tiene una estructura similar (Veloso, 2004: 105). A pesar de ello, existe una visión más positiva relativa al Congreso, y al rol que podría desempeñar en el PMP.

"All the backgrounds and information indicate that the Chilean National Congress will be able to have more proactive role in foreign policies and national defense, without infringing upon the prerogatives of the executive power" (Valdivieso, 2009: 29). "Members of parliament have the legal mechanism, the administratives and the statutory to access the relevant information on foreign affairs and national defense" (Valdivieso, 2009: 29). 
EL ROL DE LA COMISIÓN DE DEFENSA DE LA CÁMARA DE DIPUTADOS EN LA FORMULACIÓN...

Tabla $N^{\circ} 2$

Perfil miembros Comisión de Defensa Nacional 2006- 2009

\begin{tabular}{|c|c|c|c|}
\hline Miembro Comisión & Partido & Profesión & $\begin{array}{l}\text { Experiencia previa en } \\
\text { Comisión de Defensa }\end{array}$ \\
\hline Iván Paredes & PS & Empresario & $\mathrm{NO}$ \\
\hline Alberto Cardemil H. & Ind. & Abogado & sí \\
\hline Sergio Correa de la Cerda & UDI & Agricultor & Sí \\
\hline María Angélica Cristi Marfil & UDI & Socióloga & Sí \\
\hline Eduardo Díaz Del Río & PRI & Licenciado en Derecho & $\mathrm{NO}$ \\
\hline Renán Fuentealba V. & PDC & Abogado & sí \\
\hline Patricio Hales D. & PPD & Arquitecto & SÍ \\
\hline Roberto León & PDC & Abogado & Sí \\
\hline Rosauro Martínez Labbé & $\mathrm{RN}$ & Agricultor & $\mathrm{NO}$ \\
\hline José Pérez Arriagada & PRSD & Técnico Agrícola & Sí \\
\hline Jorge Tarud D. & PPD & Ejercicio libre & Sí \\
\hline Jorge Ulloa. A. & UDI & Profesor de Historia & $\mathrm{NO}$ \\
\hline Ignacio Urrutia B. & UDI & Agricultor & NO \\
\hline
\end{tabular}

Fuente: Elaboración propia en base a la información disponible en www.bcn.cl

La Tabla 2 muestra cierto indicador de especialización en materia de defensa de los diputados integrantes de la Comisión en el periodo 2006- 2010. Como se aprecia, 8 de los 13 integrantes de esa instancia tenían experiencia previa en la Comisión de Defensa. Tal como lo reconoce la literatura, la permanencia en una comisión es un poderoso indicador de la capacidad de los legisladores para influir en el PMP. Ello, tanto por su especialización en materia de defensa como por la mayor "fluidez" de los intercambios con el sector defensa (Ejecutivo, burocracia, FF.AA.).

¿Qué actividades desarrolla principalmente la Comisión de Defensa de la cámara baja? La revisión de las actas de sesión de la Comisión entre 2006 y 2009 da cuenta del conjunto de tareas en las que concentra su trabajo esta instancia especializada. Como se aprecia en la Tabla $N^{0} 3$, el trabajo de la Comisión puede agruparse en tres categorías: legislativa, fiscalización, intercambios con la sociedad civil y relaciones cívico-militares. La subcategoría "materia legislativa" muestra una alta frecuencia dentro de las actividades que desarrolla la Comisión en el periodo 2006-2010, junto con "estudio legislativo". Ahora, la cantidad de proyectos de ley tramitados por la Comisión en este periodo no es muy alta. Solo 4 mensajes y 7 mociones fueron tratados en la tabla de la Comisión. 
Tabla No 3

Frecuencia de actividades Comisión de Defensa Cámara de Diputados

\begin{tabular}{|c|c|c|c|}
\hline Legislativa & Fiscalización & $\begin{array}{l}\text { Intercambio sociedad } \\
\text { civil }\end{array}$ & $\begin{array}{l}\text { Relaciones cívico } \\
\text { militares }\end{array}$ \\
\hline $\begin{array}{l}\text { - Materia Legislativa } \\
(60) \\
\text { - Estudio Legislativo } \\
\text { (5) } \\
\text { - Mensaje tramitados } \\
\text { por la Comisión (4) } \\
\text { - Mociones tratadas } \\
\text { por la Comisión (7) } \\
\text { - Aprobación } \\
\text { proyecto de ley (6) } \\
\text { - Aprobaciones } \\
\text { legislativas parciales } \\
\text { (2) }\end{array}$ & $\begin{array}{l}\text { - Citación } \\
\text { Subsecretaría de } \\
\text { Carabineros (1) } \\
\text { - Subsecretaría Policía } \\
\text { Civil (1) } \\
\text { - Oficio Legislativo- } \\
\text { Ejecutivo (24) } \\
\text { - Oficio Ejecutivo- } \\
\text { Legislativo (47) } \\
\text { - Invitación a otras } \\
\text { autoridades del Estado } \\
\text { de Chile (no gabinete) } \\
\text { (28) } \\
\text { - Oficio Comisión- } \\
\text { Contraloría (1) } \\
\text { - Requiere } \\
\text { información a las } \\
\text { FF.AA. (25) } \\
\text { - Creación comisión } \\
\text { investigadora (1) }\end{array}$ & $\begin{array}{l}\text { - Jornada Temática (1) } \\
\text { - Audiencia Pública } \\
\text { (1) } \\
\text { - Invitación Think } \\
\text { Tanks (4) } \\
\text { - Intercambio } \\
\text { Internacional (7) }\end{array}$ & $\begin{array}{l}\text { - Citaciones al } \\
\text { Ministro de Defensa } \\
\text { (18) } \\
\text { - Citación } \\
\text { Subsecretaría de } \\
\text { Guerra (11) } \\
\text { - Citación } \\
\text { Subsecretaría de } \\
\text { Marina (1) } \\
\text { - Invitación } \\
\text { Comandantes en Jefe } \\
\text { (16) }\end{array}$ \\
\hline
\end{tabular}

Fuente: Elaborado a partir de la información disponible en www.camara.cl

En cuanto a la categoría "fiscalización", la Comisión realizó múltiples acciones tendientes a fiscalizar al sector defensa y las FF.AA. Ello, se materializa a través del envío frecuente de oficios al Ministerio de Defensa, a las respectivas FF.AA., invitaciones a declarar ante la Comisión a diversas autoridades, funcionarios o asesores competentes sobre materias conocidas por la Comisión. Los intercambios con la sociedad civil son otro campo de acción de la Comisión. Esto último se materializa a través de la figura de las Jornadas Temáticas, las Audiencias Públicas e invitaciones a centros de estudios o think tanks para que manifiesten su opinión ante la Comisión o presenten información relevante relativa a proyectos de ley vistos por esta o bien para intercambiar opiniones y visiones sobre aspectos relativos al sector defensa que puedan ser de interés para la denominada "comunidad de defensa". Una última categoría relevante en la que es posible agrupar el trabajo de la Comisión, corresponde a las relaciones civil-militares, traducida en invitaciones a autoridades 
EL ROL DE LA COMISIÓN DE DEFENSA DE LA CÁMARA DE DIPUTADOS EN LA FORMULACIÓN...

del sector defensa, jefes castrenses y otros actores. Ello, con el objeto de intercambiar pareceres y opiniones sobre hechos ocurridos o bien sobre materias legislativas tramitadas en la comisión.

\section{Conclusiones}

Las comisiones de defensa de ambas cámaras no solo proponen a la sala la aprobación o rechazo de los proyectos de ley que se someten a su conocimiento en general y en particular, la aprobación presupuestaria anual en las partidas de defensa, la entrada de tropas extranjeras al territorio o la salida de tropas nacionales e incluso la declaración de estado de asamblea en caso de guerra. Como se pretende desarroIlar en la investigación, las comisiones de defensa nacional juegan un papel clave en la fiscalización de los procesos administrativos y el devenir de cada una de las reparticiones del Ministerio de la Defensa Nacional, como asimismo de cada uno de los servicios que componen las Fuerzas Armadas y de Orden en el caso de la Cámara de Diputados y en la formulación de las políticas de defensa.

\section{Referencias}

Aleman, Eduardo; Pachon, Mónica. 2008. "Las Comisiones de conciliación en los procesos legislativos en Chile y Colombia". Política y Gobierno XV (1): 3-37.

Aninat, Cristóbal; Londregan, John; Navia, Patricio; Vial, Joaquín. 2006. "Political institutions, policymaking processes and policy outcomes in Chile". Research Network Working Paper R- 521, IADB, Latin American Research Network.

AnINAt, Cristóbal. 2004. "Balance de poderes legislativos en Chile: ¿Presidencialismo exagerado o base de un sistema político cooperativo?" Política 47 (2): 127-148.

Berríos, Fabiola; Gamboa, Ricardo. 2006. "El Congreso Nacional chileno y el ejercicio de sus funciones legislativa y fiscalizadora". Política 47 (2): 99-126.

BejAR, LuIs. 2007. "La representación parlamentaria en América Latina: las comisiones permanentes y los partidos en el Congreso". América Latina Hoy 43: 107-122.

Boeninger, Edgardo. 2007. Políticas Públicas en Democracia. Institucionalidad y experiencia chilena. Uqbar Editores: Santiago.

BORN, Hans. 2002. "Learning for best practices of parliamentary oversight of the security sector", Working Paper № 1, Geneva, Center of Democratic Control of Armed Forces.

Born, HAns; FLury, P.; LUNN, SIMON. 2003. Oversight and Guidance: the relevance of parliamentary oversight of security sector and its reform. DCAF Document 4, Brusselas, Geneva. 
Bruneau, Thomas. 2005. "Civil-Military Relations in Latin America: The Hedgehog and the Fox Revisited". Fuerzas Armadas y Sociedad 19 (1): 111-131.

Bruneau, Thomas; Matel, Fiorina. 2008. "Towards a new conceptualisation of democratization and Civil- Military relations". Democratization 15 (5): 909-927.

Bruneau, Thomas; Matel, Cristiana; Sakoda, Sak. 2009. "National Security Councils: their potential functions in democratic civil- military relations". Defense and Security Analysis 25 (3): 255- 269.

Carey, John. 2002. "Parties, coalition and the chilean Congress in the 1990s". En Morgenstern, S.; Nacif, B. (editores): Legislative Politics in Latin America. Cambridge University Press: 222-253.

Celi, Pablo. 2003. Nuevas tendencias en seguridad y defensa en América Latina. Atlas Comparativo de la Defensa en América Latina. Resdal, Buenos Aires, Argentina.

Cox, Gary; Morgenstern, Scott. 2002. "Epilogue: Latin America reactive assamblies and proactive Presidents". En Morgenstern, S.; Nacif, B. (editores): Legislative Politics in Latin America. Cambridge University Press: 446-468.

García, Mercedes; Sanchez, Francisco. 2002. "Las comisiones legislativas en América Latina: una clasificación institucional y empírica". WP 212, Institut de Ciencies Politiques i Socials, Barcelona.

Ferraro, Agustín. 2008. "Friends in High Places: Congressional Influence on the Bureaucracy in Chile". Latin American Politics and Society 50 (2): 102-129.

Follietti, Gilda; Tibilettı, Paz. 2004. Parlamento y defensa en América Latina: El papel de las comisiones. Vol. I: Argentina, Brasil, Chile y Uruguay. Resdal, Buenos Aires, Argentina.

Fuentes, Claudio. 2008. "Construyendo supremacía civil: el difícil test del control civil sobre las Fuerzas Armadas en el Cono Sur". Documento de Trabajo, CLACSO. www.bibliotecavirtualclacso.org.ar

Huneeus, Carlos; Berríos, Fabiola. 2004. "El Congreso Nacional en un régimen presidencial. El caso de Chile". Revista de Derecho Público 66: 61-95.

KINGDON, J. 1995. Agendas, alternatives and public policies. Addison Wesley: NY.

Lahera, Eugenio. 2002. Introducción a las Políticas Públicas. Fondo de Cultura Económica, México D.F.

Libro de la Defensa Nacional de Chile. 2010. Ministerio de Defensa Nacional.

Morgenstern, Scott. 2002. "Explaining Legisltive Politics in Latin America". En Morgenstern, S.; Nacif, B. (editores): Legislative Politics in Latin America. Cambridge University Press: 413-445.

Payne, J. Mark; Zovatto, Daniel; Carrillo, Fernando; Allamand, Andrés. 2003. La política importa. Democracia y desarrollo en América Latina. Washington D.C: BID. 
EL ROL DE LA COMISIÓN DE DEFENSA DE LA CÁMARA DE DIPUTADOS EN LA FORMULACIÓN...

Peters, Guy. 2003. "La capacidad para gobernar; ¿Retrocediendo hacia el centro?" Revista del CLAD 27: Caracas: 7-28.

Pion-Berlin, David; Trinkunas, Harold. 2001. "Attention Deficits: Why Politicians Ignore Defense Policy in Latin America", Latin American Research Review 42.3, 2007.

Polloni, Marco; Contreras, Arturo; Ortiz, Claudio. 2001. "Relaciones entre las Fuerzas Armadas y el Poder Legislativo en Chile: Análisis y proposiciones". Security and Defense Studies Review, Vol. 1, № 1, Spring 2001.

RIAL, JuAN. 2004. Los cambios relacionados con el Estado Nación y su influencia sobre las Fuerzas Armadas. Simposio Defensa, Sociedad y Desarrollo. Buenos Aires, Argentina.

Rivera, José Antonio. 1998. "Las comisiones en las legislatures: su papel en las políticas públicas". Política y Gobierno 5 (2): 522-556.

Red Resdal. Parlamento y Defensa en América Latina: el Papel de las Comisiones. National Democracy Endowment, Buenos Aires, Argentina.

Schmidt Hebbel, Klaus. 2007. "Chile's Growth and development: Leadership, Policy Making Process, Policies, and Results". Paper preparado for The Comission on Growth and Development. Disponible en www.iadb.org

Siavelis, Peter. 2001. "Chile: las relaciones entre el poder ejecutivo y el poder legislativo después de Pinochet". En Lanzaro, J. (ed.) Tipos de Presidencialismo y coaliciones políticas en América Latina, CLACSO: Buenos Aires.

Siavelis, 2002. "Exaggerated Presidentialism and moderate Presidents: executive-legislative relations in Chile". En Morgenstern, S.; Nacif, B. (editores): Legislative Politics in Latin America. Cambridge University Press: 79-113.

Stein, Ernesto; Tomması, Mariano. 2006. "La Política de las Políticas Públicas". Política y Gobierno 13 (2): 393-416.

Stein, Ernesto (editor). 2006. La Política de las Políticas Públicas: Progreso Económico y Social en América Latina. BID: Washington.

USAID. 2005. Handbook of Legislative Strengthening. Center for Democracy and Governance, Washington DC.

Van Eekelen, Willem F. 2005. The Parliamentary Dimension of Defence Procurement: Requirements, Production, Cooperation and Acquisition; Geneva Centre for the Democratic Control of Armed Forces Occasional Paper-5.

Veloso, David. 2004. Chile: Evaluación del Funcionamiento de la Comisión de Defensa del Parlamento. Parlamento y defensa en América Latina: El papel de las Comisiones. Vol. I: Argentina, Brasil, Chile y Uruguay. Red Resdal, Buenos Aires, Argentina. 
Valdivieso, PAtricio. 2009. "Congreso Nacional y política exterior chilena: estado actual y algunas propuestas". Estudios Internacionales 148: 147-176.

Weeks, Gregory. 2006. "Inching Toward Democracy: President Lagos and the Chilean Armed Forces", In Silvia Borzutzky and Lois Hecht Oppenheim (eds.). After Pinochet: The Chilean Road to Democracy and the Market, Gainesville: University Press of Florida: 26-41.

Weyland, Kurt. 2002. "Limitations of rational choice institutionalism for the study of Latin American Politics". Studies in Comparative International Development 37 (1): 57-85. 